\title{
Nonlinear Feedback Design for Fixed-Time Stabilization of Linear Control Systems
}

\author{
Andrey Polyakov
}

\begin{abstract}
Nonlinear control algorithms of two types are presented for uncertain linear plants. Controllers of the first type are stabilizing polynomial feedbacks that allow to adjust a guaranteed convergence time of system trajectories into selected neighborhood of the origin independently on initial conditions. The control design procedure uses block control principles and finite-time attractivity properties of polynomial feedbacks. Controllers of the second type are modifications of the second order sliding mode control algorithms. They provide global finite-time stability of the closed-loop system and allow to adjust a guaranteed settling time independently on initial conditions. Control algorithms are presented for both single-input and multi-input systems. Theoretical results are supported by numerical simulations.
\end{abstract}

\section{Index Terms}

finite-time stability, polynomial feedback, second order sliding mode control

\section{INTRODUCTION}

Finite-time stability and stabilization problems have often been a subject of research [13], [4], [19], [21]. The control theory provides many systems that exhibit finite-time convergence to the equilibrium. Frequently such systems appear in observation problems when finite-time convergence of the observed states to the real ones is required [3]. The high order sliding mode control algorithms also provide finite-time convergence to the origin [16], [20], [17], [22]. Typically such controllers have mechanical and electromechanical applications [2], [11], [5].

A. Polyakov is with Non-A INRIA - LNE, Parc Scientifique de la Haute Borne 40, avenue Halley Bat.A, Park Plaza 59650 Villeneuve d'Ascq, France, andrey.polyakov@inria.fr 
The paper deals with an extension global finite-time stability concept that is related to possible predefining of guaranteed convergence(settling) time independently on initial conditions. The corresponding property is called in this paper by fixed-time stability. Such phenomenon was discovered in [18],[10],[23],[6], where observers with predetermined finite convergence time have been developed. The present paper mostly addresses the control design problem for linear plants providing fixed-time convergence to the given set. The developed design procedure requires only controllability of the system, i.e. $\operatorname{rank}\left[B, A B, \ldots, A^{n-1} B\right]=n$. Some controllers are presented for fixed-time stabilization of the origin. These results are restricted by the case $\operatorname{rank}[B, A B]=n$.

The controls considered in this paper basically have polynomial forms. Polynomial state feedback control systems have considerable attention in nonlinear control [8]. This class of control systems appears in models of a wide range of applications such as chemical processes, electronic circuits and mechatronics, biological systems etc. This paper studies a special property of polynomial feedbacks, which is expressed in fixed-time attraction of solutions of the closedloop system into any selected neighborhood of the origin.

Usually finite-time stability is closely related with homogeneity property of the system. While being asymptotically stable and homogeneous of negative degree, the system is shown to approach the equilibrium point in finite time [16], [20]. The concept of homogeneity in bi-limit introduced in [1] generalizes this property providing that an asymptotically stable system is fixedtime stable if it is homogeneous of negative degree in 0-limit and homogeneous of positive degree in $\infty$-limit. Unfortunately, homogeneous approach does not allow to adjust or even estimate the settling time. To overcome this problem the paper introduces a special modification of the socalled "nested" (terminal) second order sliding mode control algorithm [17] that provides fixedtime stability of the origin and allows to adjust the global settling time of the closed-loop system.

All control algorithms presented in the paper are robust with respect to system disturbances and plant parameters variations in the case when the, so-called, matching condition [24] holds, that is, to guarantee successful elimination of system uncertainties or external disturbances they should act in the same subspace as an admissible control.

The paper is organized as follows. The next section discuses finite-time and fixed-time stability concepts. It also extends Lyapunov Function Method to fixed-time stability case. Section 3 states the main problem. Sections 4 and 5 consider control design algorithms for single-input and multiinput systems. Finally, numerical simulations examples and conclusions are presented. 


\section{FINITE-TIME STABILITY AND SOME FURTHER EXTENSIONS}

Consider the following system

$$
\dot{x}=g(t, x), \quad x(0)=x_{0}
$$

where $x \in \mathbb{R}^{n}$ is the vector of system states, $g: \mathbb{R}_{+} \times \mathbb{R}^{n} \rightarrow \mathbb{R}^{n}$ is a nonlinear function. The case of discontinuous right-hand sides of the system (1) is not excluded, so solutions of (1) are understood in the sense of Filippov [12]. Assume that the system (1) has zero equilibrium point.

Definition 1 ([4], [20]). The equilibrium point $x=0$ of the system (1) is said to be globally finite-time stable if it is globally asymptotically stable and any solution $x\left(t, x_{0}\right)$ of (1) reaches the equilibria at some finite time moment, i.e. $x\left(t, x_{0}\right)=0, \forall t \geq T\left(x_{0}\right)$, where $T: \mathbb{R}^{n} \rightarrow \mathbb{R}_{+} \cup\{0\}$ is the so-called settling-time function.

The finite-time stability property may demonstrate homogeneous systems with negative degree [4], [20] or the so-called sliding homogeneous systems [16]. For example, any solution of the system $\dot{x}=-x^{-1 / 3}, x \in \mathbb{R}$ converges to zero equilibrium point in finite time $T\left(x_{0}\right):=\frac{2}{3} \sqrt[3]{\left|x_{0}\right|^{2}}$.

Definition 2. The equilibrium point $x=0$ of the system (1) is said to be globally fixed-time stable if it is globally finite-time stable and the settling-time function $T\left(x_{0}\right)$ is bounded by some positive number $T_{\max }>0$, i.e. $T\left(x_{0}\right) \leq T_{\max }$ for $\forall x_{0} \in \mathbb{R}^{n}$.

For example, the system $\dot{x}=-x^{1 / 3}-x^{3}, x \in \mathbb{R}$ has the fixed-time stable zero equilibrium. Any solution $x\left(t, x_{0}\right)$ of this system converges to the origin in a finite-time and for any $x_{0} \in \mathbb{R}$ the equality $x\left(t, x_{0}\right)=0$ holds for all $t \geq 2.5$.

In the paper [6] the closed-loop control systems that demonstrate dynamics described in Definition 2 was called by "uniformly finite-time convergent". The term "stability" is used here just because this notion is more traditional and natural for describing the referred properties of the system's equilibrium points, but the term "uniformly finite-time stable" can not be used for this purpose, since it has different natural meaning discussed in [20].

The fixed-time stability can be simply extended to the case of attractive set.

Definition 3. The set $M$ is said to be globally finite-time attractive for the system (1) if any solution $x\left(t, x_{0}\right)$ of $(1)$ reaches $M$ in some finite time moment $t=T\left(x_{0}\right)$ and remains there, i.e. $x\left(t, x_{0}\right) \in M, \forall t \geq T\left(x_{0}\right)$, where $T: \mathbb{R}^{n} \rightarrow \mathbb{R}_{+} \cup\{0\}$ is the settling-time function. 
Definition 4. The set $M$ is said to be globally fixed-time attractive for the system (1) if it is globally finite-time attractive and the settling-time function $T\left(x_{0}\right)$ is bounded by some positive number $T_{\max }>0$, i.e. $T\left(x_{0}\right) \leq T_{\max }$ for $x_{0} \in \mathbb{R}^{n}$.

The property of global finite-time attractivity may demonstrate even some linear systems, but the only nonlinear ones may have fixed-time attractive sets.

Denote by $D^{*} \varphi(t)$ the upper right-hand derivative of a continuous function $\varphi(t)$, i.e. $D^{*} \varphi(t):=$ sup $\lim _{h \rightarrow+0} \frac{\varphi(t+h)-\varphi(t)}{h}$. The following simple result extends the Lyapunov function method providing the background for the fixed-time stability analysis.

Lemma 1. If there exists a continuous radially unbounded function $V: \mathbb{R}^{n} \rightarrow \mathbb{R}_{+} \cup\{0\}$ such that 1) $V(x)=0 \Rightarrow x \in M$; 2) any solution $x(t)$ of (1) satisfies the inequality $D^{*} V(x(t)) \leq$ $-\left(\alpha V^{p}(x(t))+\beta V^{q}(x(t))\right)^{k}$ for some $\alpha, \beta, p, q, k>0: p k<1, q k>1$ then the set $M \subset \mathbb{R}^{n}$ is globally fixed-time attractive for the system (1) and $T\left(x_{0}\right) \leq \frac{1}{\alpha^{k}(1-p k)}+\frac{1}{\beta^{k}(q k-1)}, \forall x_{0} \in \mathbb{R}^{n}$.

Proof: Due to 2) we have $D^{*} V(x(t)) \leq-\alpha^{k} V^{p k}(x(t))$ if $V(x(t)) \leq 1$ and $D^{*} V(x(t)) \leq$ $-\beta^{k} V^{q k}(x(t))$ for $V(x(t))>1$. Hence, for any $x(t)$ such that $V(x(0))>1$ the last inequality guarantees $V(x(t)) \leq 1$ for $t \geq \frac{1}{\beta^{k}(q k-1)}$ and for any $x(t)$ such that $V\left(x\left(t_{0}\right)\right) \leq 1$ we derive $V(x(t))=0$ for $t \geq t_{0}+\frac{1}{\alpha^{k}(1-p k)}$. Therefore, $V(x(t))=0$ for all $t \geq \frac{1}{\alpha^{k}(1-p k)}+\frac{1}{\beta^{k}(q k-1)}$ and any solution $x(t)$ of (1). Applying the condition 1) we finish the proof of this lemma.

Replacement of the condition 1) in Lemma 1 by $V(x)=0 \Leftrightarrow x=0$ helps to check invariance of the set $M$. So, for $M=\{0\}$ Lemma 1 allows to analyze the fixed-time stability of the origin.

\section{Problem Statement and Basic Assumptions}

Consider the control system of the form

$$
\dot{x}=A x+B u+f(t, x)
$$

where $x \in \mathbb{R}^{n}$ is the vector of system states, $A \in \mathbb{R}^{n \times n}$ is the system matrix, $B \in \mathbb{R}^{n \times m}$ is the matrix of control gains, $u \in \mathbb{R}^{m}$ is the vector of control inputs, and the function $f: \mathbb{R}_{+} \times \mathbb{R}^{n} \rightarrow$ $\mathbb{R}^{n}$ describes systems uncertainties.

We study the system (2) under the following classical assumptions: 1. The pair $(A, B)$ is controllable, i.e. $\operatorname{rank}\left[B, A B, A^{2} B, \ldots, A^{n-1} B\right]=n$. 2. The uncertain function $f(t, x)$ satisfies the so-called matching condition (see, for example, [24]), i.e. $f(t, x)=B \gamma(t, x)$, where $\gamma(t, x)$ ) 
is an unknown but bounded function. The function $\gamma(t, x)$ is assumed to be bounded by some known function $\gamma_{0}(t, x) \geq 0$, i.e. $\|\gamma(t, x)\|_{\infty} \leq \gamma_{0}(t, x)$ for $\forall t>0$ and $\forall x \in \mathbb{R}^{n}$.

Denote a closed ball of radius $r>0$ with the center in the origin by $B_{r}$, i.e. $B_{r}:=\left\{x \in \mathbb{R}^{n}\right.$ : $\left.\|x\|_{\infty} \leq r\right\},\|x\|_{\infty}:=\max _{i=1,2, \ldots, n}\left|x_{i}\right|$.

This paper address two following problems:

- to design a feedback control $u=u(t, x)$ for the system (2), which provides the fixed-time attractivity property of the given ball $B_{r}$ for a predefined global settling-time estimate $T_{\max }$;

- to design a feedback control $u=u(t, x)$, which guarantees fixed-time stability of the origin of the closed-loop system (2) for a predefined global settling-time estimate $T_{\max }$.

\section{Fixed-Time Controllers FOR Single InPUT Systems}

\section{A. Fixed-time Attractivity}

Consider the case $m=1$. Then the linear transformation $y=G x, G=\left[A^{n-1} b, A^{n-2} b, \ldots, A b, b\right]^{-1}$ brings (may be after reordering) the initial system (2) to the Brunovsky form

$$
\dot{y}_{1}=y_{2}, \quad \dot{y}_{2}=y_{3}, \quad \ldots, \quad \dot{y}_{n}=\sum_{i=1}^{n} a_{i} y_{i}+u(y)+\gamma(t, y)
$$

where $a_{i} \in \mathbb{R}$ and the uncertain term $\gamma$ is described in the previous section, $\|\gamma(t, y)\|_{\infty}<\gamma_{0}(t, y)$.

Introduce the nonlinear coordinate transformation $s=\Phi(y)$ by the following formulas:

$$
s_{i}=y_{i}+\varphi_{i}, \quad i=1,2, \ldots, n, \quad \varphi_{1}=0, \quad \varphi_{i+1}=\alpha_{i}\left(y_{i}+\varphi_{i}\right)+\beta_{i}\left(y_{i}+\varphi_{i}\right)^{3}+\sum_{k=1}^{i} \frac{\partial \varphi_{i}}{\partial y_{k}} y_{k+1},
$$

where $\alpha_{i}, \beta_{i}, i=1,2, \ldots, n-1$ are some positive numbers. The presented coordinate transformation is smooth and nonsingular. The inverse transformation $y=\Phi^{-1}(s)$ is defined as follows:

$$
y_{i}=s_{i}+\psi_{i}, \quad i=1,2, \ldots, n, \quad \psi_{1}=0, \quad \psi_{i+1}=-\alpha_{i} s_{i}-\beta_{i} s_{i}^{3}+\sum_{k=1}^{i} \frac{\partial \psi_{i}}{\partial s_{k}}\left(-\alpha_{k} s_{k}-\beta_{k} s_{k}^{3}+s_{k+1}\right) .
$$

Formal calculations show that the system (3) is equivalent to

$$
\dot{s}_{1}=-\alpha_{1} s_{1}-\beta_{1} s_{1}^{3}+s_{2}, \quad \dot{s}_{2}=-\alpha_{2} s_{2}-\beta_{2} s_{2}^{3}+s_{3}, \quad \ldots, \quad \dot{s}_{n}=\sum_{i=1}^{n} a_{i} y_{i}+\sum_{k=1}^{n-1} \frac{\partial \varphi_{n}}{\partial y_{k}} y_{k+1}+u+\gamma(t, y)
$$

and the feedback control of the form

$$
u=-\alpha_{n} s_{n}-\beta_{n} s_{n}^{3}-\sum_{i=1}^{n} a_{i} y_{i}-\sum_{k=1}^{n-1} \frac{\partial \varphi_{n}}{\partial y_{k}} y_{k+1}, \quad \text { where } s=\Phi(y)
$$

provides fixed-time attractivity property of the closed-loop system (4). The next lemma gives a background for an appropriate adjustment of the control parameters $\alpha_{i}$ and $\beta_{i}$. 
Lemma 2. Let 1) $\varepsilon_{i}>0, i=1,2, \ldots, n$ and $T_{\max }>0$ are some positive numbers; 2) $\alpha_{1}=\bar{\alpha}_{1}+\frac{\varepsilon_{2}}{\varepsilon_{1}}$, $\ldots, \alpha_{n-1}=\bar{\alpha}_{n-1}+\frac{\varepsilon_{n}}{\varepsilon_{n-1}}, \alpha_{n}:=\alpha_{n}(t, y)=\bar{\alpha}_{n}+\frac{\gamma_{0}(t, y)}{\varepsilon_{n}}$, where $\bar{\alpha}_{i}$ are nonnegative numbers and $\|\gamma(t, y)\|_{\infty}<\gamma_{0}(t, y)$; 3) $\beta_{i} \geq \frac{\bar{\alpha}_{i} / \varepsilon_{i}^{2}}{\exp \left(2 \bar{\alpha}_{i} \frac{T_{\max }}{n}\right)-1}, i=1,2, \ldots, n$. Then any solution $s(t)$ of the system (4)-(5) satisfies the inequalities $\left|s_{i}(t)\right| \leq \varepsilon_{i}$ for $\forall t \geq T_{\max }$.

Proof: Denote $V_{i}(t)=\left|s_{i}(t)\right|, i=1,2, \ldots, n$. For all $t>0$ the inequality $D^{*} V_{n}(t) \leq$ $-\alpha_{n} V_{n}(t)-\beta_{n} V_{n}^{3}(t)+\gamma_{0}$ holds. Hence, $\dot{V}_{n}(t) \leq-\bar{\alpha}_{n} V_{n}(t)-\beta_{n} V_{n}^{3}(t)$ for $t>0: V_{n}(t) \geq$ $\varepsilon_{n}$. Studying the last inequality we derive $\frac{\beta_{n}}{\bar{\alpha}_{n}} V_{n}^{2}(t)+1 \leq\left(1-\frac{\beta_{n} V_{n}^{2}(0)}{\beta_{n} V_{n}^{2}(0)+\bar{\alpha}_{n}} e^{-2 \bar{\alpha}_{n} t}\right)^{-1}$. Hence, $V_{n}(t) \leq \varepsilon_{n}$ for all $t \geq T_{\max } / n$. The similar considerations for $i=n-1$ give $V_{i}(t) \leq \varepsilon_{i}$ for all $t \geq(n-i+1) T_{\max } / n$. The last step $(i=1)$ provides $\left|s_{i}(t)\right|<\varepsilon_{i}$ for all $t>T_{\max }$.

Corollary 1. Selection of the parameters $\varepsilon_{i}=\varepsilon>0$ and $\bar{\alpha}_{i}=1$ for $i=1,2, \ldots, n$ under conditions of Lemma 2 implies the inequality $\|s(t)\|_{\infty} \leq \varepsilon$ for all $t>T_{\max }$, where $s(t)$ is an arbitrary solution of the system (4)-(5) with control parameters $\alpha_{i}=2, i=1,2, \ldots, n-1$; $\alpha_{n}(t, x)=1+\gamma_{0}(t, x) / \varepsilon$ and $\beta_{i}=\beta:=\varepsilon^{-2}\left(\exp \left(\frac{2 T_{\max }}{n}\right)-1\right)^{-1}, i=1,2, \ldots, n$.

The presented corollary shows the way for designing of the control, which provides the fixedtime attractivity property of the ball $B_{\varepsilon}$ for the system (4). The radius of the ball $\varepsilon$ and the global settling-time $T_{\max }$ can be selected in arbitrary way. Show now that the designed controller will guarantee the same fixed-time attractivity property for the original system (3).

Denote a set of all polynomials of the order $k$ by $\mathbb{P}^{k}$.

Corollary 2. If conditions of Corollary 1 hold, then there exist polynomials $p_{i} \in \mathbb{P}^{i}, i=$ $1,2, \ldots, n$ with nonnegative coefficients such that $\left|y_{i}(t)\right| \leq p_{i}(q) \varepsilon, \forall t>T_{\max }$, where $q=$ $\left(\exp \left(\frac{2 T_{\max }}{n}\right)-1\right)^{-1}$ and $y(t)=\left(y_{1}(t), \ldots, y_{n}(t)\right)^{T}$ is an arbitrary solution of the system (3), (5).

Proof: Due to Corollary 1 we have that $\left|s_{i}\right| \leq \varepsilon$ for all $t>T_{\max }$. Let $z_{i}>0, i=$ $1,2, \ldots, n$ and $\tilde{\psi}_{1}=0, \tilde{\psi}_{i+1}=\tilde{\psi}_{i+1}\left(z_{1}, z_{2}, \ldots, z_{i+1}\right):=2 z_{i}+\beta z_{i}^{3}+\sum_{k=1}^{i} \frac{\partial \tilde{\psi}_{i}}{\partial z_{k}}\left(2 z_{k}+\beta z_{k}^{3}+z_{k+1}\right)$. It is easy to see that $\left|\psi_{i}\left(s_{1}, \ldots, s_{i}\right)\right| \leq \tilde{\psi}_{i}\left(\left|s_{1}\right|, \ldots,\left|s_{i}\right|\right)$. Show now that $\tilde{\psi}_{i}(\varepsilon, \ldots, \varepsilon)=\varepsilon \sum_{j=0}^{i-1} \mu_{i j}\left(\beta \varepsilon^{2}\right)^{j}$, where $\mu_{i j}$ are some nonnegative numbers. Obviously, $\tilde{\psi}_{1}=0$ and $\tilde{\psi}_{2}=2 z_{1}+\beta z_{1}^{3}$ satisfy the required representation. Assume that it fulfils for $\tilde{\psi}_{i}$ and show the same property for $\tilde{\psi}_{i+1}$. Due to recursive polynomial structure of $\tilde{\psi}_{i}$ we conclude that for $z_{j}=\varepsilon, j=1,2, \ldots, i \frac{\partial \tilde{\psi}_{i}\left(z_{1}, \ldots, z_{i}\right)}{\partial z_{k}}=\sum_{j=0}^{i-1} \hat{\mu}_{i j}^{k}\left(\beta \varepsilon^{2}\right)^{j}$, where $\hat{\mu}_{i j}^{k}$ are some 
nonnegative numbers. Hence, $\tilde{\psi}_{i+1}(\varepsilon, \ldots, \varepsilon)=\varepsilon\left(1+\beta \varepsilon^{2}\right)+\varepsilon \sum_{k=1}^{i} \sum_{j=0}^{i-1} \hat{\mu}_{i j}^{k}\left(\beta \varepsilon^{2}\right)^{j}\left(2+\beta \varepsilon^{2}\right)=\varepsilon \sum_{j=0}^{i} \mu_{i+1 j}\left(\beta \varepsilon^{2}\right)^{j}$ and the required property is proven. Finally taking into account $\beta \varepsilon^{2}=q$ and $\left|y_{i}(t)\right| \leq\left|s_{i}(t)\right|+$ $\left|\psi_{i}\left(s_{1}(t), \ldots, s_{i}(t)\right)\right| \leq p_{i}(q) \varepsilon$, where $p_{i}(q):=1+\sum_{j=0}^{i-1} \mu_{i j} q^{j}$, we finish the proof.

Remark that the proof of Corollary 2 gives an algorithm of construction of the polynomials $p_{i}(q) \in \mathbb{P}^{i}$ introduced in its statement. These polynomials are needed for adjustment of control parameters to guarantee convergence of all solutions of the original system (2) into the required neighborhood of the origin. The explicit form of the corresponding polynomial can be obtained using the recursive formulas for $\tilde{\psi}_{i}$ calculated in symbolic computation packages such as Mathematica or Symbolic Toolbox of MATLAB. In particular the first three polynomials have the form: $p_{1}(q)=1, p_{2}(q)=3+q, p_{3}(q)=9+12 q+3 q^{2}$.

Taking into account $p_{i}(q) \leq p_{i+1}(q)$ we may formulate the theorem.

Theorem 1. If $m=1$ and the control $u$ has the form (5) with parameters $\alpha_{i}=2, i=1,2, \ldots, n-1$, $\alpha_{n}(t, x)=1+\gamma_{0}(t, x) / \varepsilon, \beta_{i}=\frac{q}{\varepsilon^{2}}, i=1,2, \ldots, n, \varepsilon=r /\left(\left\|G^{-1}\right\|_{\infty} p_{n}(q)\right), q=\left(\exp \left(\frac{2 T_{\max }}{n}\right)-1\right)^{-1}$, where $r>0$ and $T_{\max }>0$ are arbitrary numbers and the polynomial $p_{n} \in \mathbb{P}^{n}$ is introduced in Corollary 2, then the ball $B_{r}$ is the globally fixed-time attractive set of the closed-loop system (2) with the settling-time function bounded by $T_{\max }$.

Theorem 1 mostly declares the theoretical possibility for designing of a nonlinear control providing fixed-time attractivity property of the closed-loop linear control system. The practical applications may require more precise adjustment of control parameters basing on Lemma 2 .

\section{B. Fixed-time stability}

The scheme presented above allows to design the control $u_{a}(t, x):=u(t, x)$ for the single input case of the system (2), which guarantees fixed-time attractivity property of an arbitrary ball $B_{r}$ with the center in the origin. The simplest combination of this controller with some control law $u_{f}(x)$ providing local finite-time stability of the origin for the system (2) gives us a hybrid control algorithm $u_{f x}(t, x)=\left\{\begin{array}{lll}u_{a}(t, x) & \text { for } & x \notin B_{r} \\ u_{f}(t, x) & \text { for } & x \in B_{r}\end{array}\right.$, which can afford fixed-time stability of the origin for the system (2). Some design procedures of local finite-time controllers can be found, for example, in [16] or [14]. However, the existed controllers providing local finite-time stability do not have precise algorithms for adjustment of control parameters to predefine the 
local settling time estimate (excepting the so-called "twisting" second order $(n=2)$ sliding mode system, see, for example, [22]). To realize the presented hybrid scheme we need to prevent the appearance of switching (chattering) regimes [9] around the boundary of the ball $B_{r}$, which may slow down or even prevent the convergence process. Therefore the "solid" (non-hybrid) controller providing fixed-time stability of the origin for the system (2) is more preferable. The designing such controller is a difficult problem, which is solved in this paper only for linear plants satisfying the additional assumption $\operatorname{rank}[B, A B]=n$. This condition definitely restricts the class of controllable systems, but it still covers a lot of real-life control systems. For example, mechanical systems with maximum numbers of control inputs [5] obviously satisfy this condition. For $m=1$ the presented assumption implies $n=2$.

Introduce the following denotation $z^{[q]}=|z|^{q} \operatorname{sign}[z], z, q \in \mathbb{R}$, which presents the involution operation without loss of number's sign. Remark, $z^{[1 / k]}=z^{1 / k}$ and $z^{[k]}=z^{k}$ for odd integers $k$.

Theorem 2. Let $T_{\max }>0$ be an arbitrary positive number, $m=1, n=2$ and the control $u(t, x)=$ $u_{f x}(t, G x), x \in \mathbb{R}^{2}$ has the form $u_{f x}(t, y)=-a_{1} y_{1}-a_{2} y_{2}-\frac{\alpha_{1}+3 \beta_{1} y_{1}^{2}+2 \gamma_{0}(t, y)}{2} \operatorname{sign}[s]-\left(\alpha_{2} s+\beta_{2} s^{[3]}\right)^{\left[\frac{1}{2}\right]}$, where $s=y_{2}+\left(y_{2}^{[2]}+\alpha_{1} y_{1}+\beta_{1} y_{1}^{[3]}\right)^{[0.5]}, 0.5 \alpha_{1}=\alpha_{2}=0.5 \beta_{1}=\beta_{2}=64 T_{\max }^{-2}$. Then the origin of the closedloop system (2) is globally fixed-time stable with the settling-time function bounded by $T_{\max }$.

Proof: I. Show that $s=0$ is a sliding surface of the closed-loop system (2). Consider the Lyapunov function candidate $V\left(y_{1}, y_{2}\right):=\left|s\left(y_{1}, y_{2}\right)\right|$ and calculate its total upper righthand Dini derivative along the trajectories of the closed-loop system (3) $D^{*} V\left(y_{1}(t), y_{2}(t)\right)=$ $\dot{y}_{2}(t) \operatorname{sign}[s(t)]+\left(\left|y_{2}(t)\right| \dot{y}_{2}(t) \operatorname{sign}[s(t)]+\frac{\alpha_{1}+3 \beta_{1} y_{1}^{2}(t)}{2} y_{2}(t) \operatorname{sign}[s(t)]\right) /\left|y_{2}^{[2]}(t)+\alpha_{1} y_{1}(t)+\beta_{1} y_{1}^{[3]}(t)\right|^{\frac{1}{2}}$ for $s \neq 0$. Taking into account the equality $\left(\alpha_{2} s+\beta_{2} s^{[3]}\right)^{\left[\frac{1}{2}\right]} \operatorname{sign}[s]=\left(\alpha_{2}|s|+\beta_{2}|s|^{3}\right)^{\frac{1}{2}}$ we obtain $\dot{y}_{2} \operatorname{sign}[s]=\left(a_{1} y_{1}+a_{2} y_{2}+u_{f x}(y)+\gamma(t, x)\right) \operatorname{sign}[s]=-\frac{\alpha_{1}+3 \beta_{1} y_{1}^{2}}{2}-\left(\alpha_{2}|s|+\beta_{2}|s|^{3}\right)^{1 / 2}-\left(\gamma_{0}-\gamma\right)$ for $s \neq 0$. Hence, we conclude that $D^{*} V \leq-\left(\alpha_{2} V+\beta_{2} V^{3}\right)^{1 / 2}$ and following the proof of Lemma 1 we guarantee $V(t):=|s(t)|=0$ for all $t \geq T_{s}:=\frac{2}{\sqrt{\alpha_{2}}}+\frac{2}{\sqrt{\beta_{2}}}=\frac{T_{\max }}{2}$.

II. The equality $s=0$ implies $2 y_{2}^{[2]}+\alpha_{1} y_{1}+\beta_{1} y_{1}^{3}=0$ and the sliding motion equation takes the form $\dot{y}_{1}=-\left(\frac{\alpha_{1}}{2} y_{1}+\frac{\beta_{1}}{2} y_{1}^{3}\right)^{[1 / 2]}$. Using Lemma 1 with the Lyapunov function $V\left(y_{1}\right)=\left|y_{1}\right|$ we show $y_{1}=0$ for all $t \geq \frac{T_{\max }}{2}+\frac{2 \sqrt{2}}{\sqrt{\alpha_{1}}}+\frac{2 \sqrt{2}}{\sqrt{\beta_{1}}}=T_{\max }$. Finally we remark that $y_{1}=0$ and $s=0$ imply $y_{2}=0$.

Remark that for $\beta_{1}=0$ the switching line $s=0$ coincides with the sliding surface of "nested" controller [17], which provides finite-time stability of the closed-loop system. The polynomial terms $\beta_{1} y_{1}^{[3]}$ and $\beta_{2} s^{[3]}$ are introduced to guarantee the fixed-time stability property. 


\section{FiXed-Time Controllers for Multi Input Systems}

\section{A. Block Decomposition}

Introduce denotations: $\operatorname{rown}(W)$ and $\operatorname{coln}(W)$ are the number of rows and columns of the matrix $W$, respectively; $\operatorname{ker}(W)$ and $\operatorname{range}(W)$ are the null space and the column space of $W$; $\operatorname{null}(W)$ is the matrix with columns defining the orthonormal basis of $\operatorname{ker}(W)$.

To adapt the fixed-time control design scheme developed for the single-input case we need to decompose the original multi input system (2) to a block from [7]. The required coordinate transformation can be constructed using supporting matrices provided by Algorithm 1.

Algorithm 1. I. Initialization : $A_{0}=A, B_{0}=B, T_{0}=I_{n}, k=0$. II. Recursion: While $\operatorname{rank}\left(B_{k}\right)<\operatorname{rown}\left(A_{k}\right)$ do $A_{k+1}=B_{k}^{\perp} A_{k}\left(B_{k}^{\perp}\right)^{T}, B_{k+1}=B_{k}^{\perp} A_{k} \tilde{B}_{k}, T_{k+1}=\left(\begin{array}{c}B_{k}^{\perp} \\ \tilde{B}_{k}\end{array}\right), k=k+1$, where $B_{k}^{\perp}:=\left(\operatorname{null}\left(B_{k}^{T}\right)\right)^{T}, \tilde{B}_{k}:=\left(\operatorname{null}\left(B_{k}^{\perp}\right)\right)^{T}$.

This algorithm can be easily realized in a computational software system such as MATLAB.

Lemma 3. If the pair $(A, B)$ is controllable then 1) Algorithm 1 terminates after finite number of steps $k \leq n-1 ; 2)$ the matrices $T_{i} \in \mathbb{R}^{\text {rown }\left(B_{i}\right) \times \operatorname{rown}\left(B_{i}\right)}, i=1,2, \ldots, k$ are orthogonal; 3) the orthogonal coordinate transformation $y=G x$

$$
G=\left(\begin{array}{cc}
T_{k} & 0 \\
0 & I_{w_{k}}
\end{array}\right)\left(\begin{array}{cc}
T_{k-1} & 0 \\
0 & I_{w_{k-1}}
\end{array}\right) \cdots\left(\begin{array}{cc}
T_{2} & 0 \\
0 & I_{w_{2}}
\end{array}\right) T_{1}, \text { where } w_{i}:=n-\operatorname{rown}\left(T_{i}\right)
$$

reduces the original system (2) to the following block form

$$
\left\{\begin{array}{l}
\dot{y}_{1}=A_{11} y_{1}+A_{12} y_{2} \\
\dot{y}_{2}=A_{21} y_{1}+A_{22} y_{2}+A_{23} y_{3} \\
\ldots \\
\dot{y}_{k}=A_{k 1} y_{1}+\ldots+A_{k k} y_{k}+A_{k k+1}(u+\gamma(t, y))
\end{array}\right.
$$

where $y=\left(y_{1}, \ldots, y_{k}\right)^{T}, y_{i} \in \mathbb{R}^{n_{i}}, n_{i}:=\operatorname{rank}\left(B_{k-i}\right), A_{k k+1}=\tilde{B}_{0} B_{0}$ and $A_{i j} \in \mathbb{R}^{n_{i} \times n_{j}}$ are blocks of the matrix $G A G^{T}$ such that $\operatorname{rank}\left(A_{i+1}\right)=n_{i}, i=1,2, \ldots, k$

Proof: I. Denote $B_{0}=B, A_{0}=A$ and $r_{0}=\operatorname{rank}\left(B_{0}\right)$. Controllability of $\left(A_{0}, B_{0}\right)$ implies $r_{0}>0$. For $r_{0}=n$ Algorithm 1 stops after initialization showing that the original system does not require transformation. II. Consider the case $r_{0}<n$. In this case $\operatorname{dim}\left(\operatorname{range}\left(B_{0}\right)\right)=r_{0}$, 
$\operatorname{dim}\left(\operatorname{ker}\left(B_{0}^{T}\right)\right)=n-r_{0}$ and $\mathbb{R}^{n}=\operatorname{range}\left(B_{0}\right) \oplus \operatorname{ker}\left(B_{0}^{T}\right)$. Columns of the matrix null $\left(B_{0}^{T}\right) \in \mathbb{R}^{n \times\left(n-r_{0}\right)}$ by definition are the orthonormal basis for $\operatorname{ker}\left(B_{0}^{T}\right) \subset \mathbb{R}^{n}$, so $\operatorname{ker}\left(B_{0}^{T}\right)=\operatorname{range}\left(\operatorname{null}\left(B_{0}^{T}\right)\right)$. Since range $\left(B_{0}\right)$ is an orthogonal complement to $\operatorname{ker}\left(B_{0}^{T}\right)$ then $\operatorname{range}\left(B_{0}\right)=\operatorname{ker}\left(\operatorname{null}\left(B_{0}^{T}\right)^{T}\right)$ and columns of the matrix $\left(B_{0}^{\perp}\right)^{T}=\operatorname{null}\left(B_{0}^{T}\right)$ together with columns of the matrix $\left(\tilde{B}_{0}\right)^{T}=\operatorname{null}\left(\operatorname{null}\left(B_{0}^{T}\right)^{T}\right)$ provide the orthonormal basis in $\mathbb{R}^{n}$. So, the block matrix $T_{1}$ is square and orthogonal. Since $B_{0}^{T} \operatorname{null}\left(B_{0}^{T}\right)=0$ then the coordinate transformation $\left(\begin{array}{cc}\tilde{y}_{1} & \tilde{y}_{2}\end{array}\right)^{T}=T_{1} x, \tilde{y}_{1} \in \mathbb{R}^{n-r_{0}}, \tilde{y}_{2} \in \mathbb{R}^{0}$ gives

$$
\dot{\tilde{y}}_{1}=\tilde{A}_{11} \tilde{y}_{1}+\tilde{A}_{21} \tilde{y}_{2}, \quad \dot{\tilde{y}}_{2}=\tilde{A}_{21} \tilde{y}_{1}+\tilde{A}_{22} \tilde{y}_{2}+\tilde{B}_{0} B_{0}(u+\gamma)
$$

where $\tilde{A}_{11}, \tilde{A}_{12}, \tilde{A}_{21}, \tilde{A}_{22}$ are the blocks of the matrix $T_{1} A T_{1}^{T}$. Obviously that $A_{11}=A_{1}$ and $A_{12}=B_{1}$, where matrices $A_{1}$ and $B_{1}$ are generated by the recursive step of Algorithm 1 .

Controllability of the pair $\left(A_{0}, B_{0}\right)$ implies controllability of $\left(A_{1}, B_{1}\right)$ [24], so we can repeat the recursive step of Algorithm 1 for $A_{1}$ and $B_{1}$. Since for each recursive step we have $\operatorname{rown}\left(A_{i+1}\right)=$ $\operatorname{rown}\left(B_{i+1}\right)=\operatorname{rown}\left(A_{i}\right)-\operatorname{rank}\left(B_{i}\right)$ and $\operatorname{rank}\left(B_{i}\right)>0$ then Algorithm 1 terminates in finite steps and the coordinate transformation $y=G x$ reduces the original system (2) to the block form (7). Finally $\operatorname{rank}\left(A_{i+1}\right)=\operatorname{rank}\left(\tilde{B}_{k-i} B_{k-i}\right)=\operatorname{rank}\left(T_{k-i} B_{k-i}\right)=\operatorname{rank}\left(B_{k-i}\right)=n_{i}$.

This paper considers the restricted robust problem statement assuming that all uncertainties and disturbances satisfy the matching condition. The control design for more general case can be done using the robustness analysis of block controllability forms presented in [15].

\section{B. Fixed-time attractivity}

Further considerations are presented for the system (2) transformed to the block form (7). Since $\operatorname{rank}\left(A_{i i+1}\right)=\operatorname{rown}\left(A_{i i+1}\right)=n_{i}$ then $A_{i+1} A_{i i+1}^{T}$ is invertible and $A_{i i+1}^{+}=A_{i i+1}^{T}\left(A_{i+1} A_{i i+1}^{T}\right)^{-1}$ is the right inverse matrix of $A_{i i+1}$. Denote $z^{[p]}=\left(z_{1}^{[p]}, \ldots, z_{k}^{[p]}\right)^{T}$ for $z=\left(z_{1}, \ldots, z_{k}\right)^{T} \in \mathbb{R}^{k}$.

By analogy to single input case introduce the nonlinear coordinate transformation $s=\Phi(y)$, $s=\left(s_{1}, \ldots, s_{k}\right)^{T}, s_{i} \in \mathbb{R}^{n_{i}}, y=\left(y_{1}, \ldots, y_{k}\right)^{T}, y_{i} \in \mathbb{R}^{n_{i}}$ by the recurrent formulas: $s_{i}=y_{i}+\varphi_{i}$, $i=1,2, \ldots, k, \varphi_{1}=0, \varphi_{i+1}=A_{i i+1}^{+}\left(\alpha_{i}\left(y_{i}+\varphi_{i}\right)+\beta_{i}\left(y_{i}+\varphi_{i}\right)^{[3]}+\sum_{j=1}^{i} A_{i j} y_{j}+\sum_{r=1}^{i} \frac{\partial \varphi_{i}}{\partial y_{r}} \sum_{j=1}^{r+1} A_{r j} y_{j}\right)$, where $\alpha_{i}>0, \beta_{i}>0$ and $i=1,2, \ldots, k-1$. The presented coordinate transformation is smooth and nonsingular. The inverse transformation $y=\Phi^{-1}(s)$ is defined as follows: $y_{i}=s_{i}+\psi_{i}$, $i=1,2, \ldots, k, \psi_{1}=0, \psi_{i+1}=A_{i+1}^{+}\left(\sum_{k=1}^{i} \frac{\partial \psi_{i}}{\partial s_{k}}\left(A_{i i+1} s_{k+1}-\alpha_{k} s_{k}-\beta_{k} s_{k}^{[3]}\right)-\alpha_{i} s_{i}-\beta_{i} s_{i}^{[3]}-\sum_{j=1}^{i} A_{i j}\left(s_{j}+\psi_{j}\right)\right)$. 
Applying the transformation $s=\Phi(y)$ to the system (7) we obtain

$$
\left\{\begin{array}{l}
\dot{s}_{1}=-\alpha_{1} s_{1}-\beta_{1} s_{1}^{3}+A_{12} s_{2} \\
\dot{s}_{2}=-\alpha_{2} s_{2}-\beta_{2} s_{2}^{3}+A_{23} s_{3} \\
\cdots \\
\dot{s}_{k}=\sum_{i=1}^{k} A_{k i} y_{i}+\sum_{i=1}^{k-1} \frac{\partial \varphi_{k}}{\partial y_{i}} \sum_{j=1}^{i+1} A_{i j} y_{j}+A_{k k+1}(u+\gamma(t, y))
\end{array}\right.
$$

So, the feedback control providing fixed-time attractivity property to the system (8) has the form

$$
u=-A_{k k+1}^{+}\left(\alpha_{k} s_{k}+\beta_{k} s_{k}^{3}+\sum_{i=1}^{k} A_{k i} y_{i}+\sum_{i=1}^{n-1} \frac{\partial \varphi_{k}}{\partial y_{i}} \sum_{j=1}^{i+1} A_{i j} y_{j}\right), s=\Phi(G y), \alpha_{k}, \beta_{k}>0
$$

Let $\varepsilon$ and $T_{\max }$ be positive numbers, $\alpha_{i}=1+\left\|A_{i+1+1}\right\|_{\infty}, i=1,2, \ldots, k-1, \alpha_{k}=1+\frac{\left\|A_{k k+1}\right\|_{\infty} \gamma_{0}(t, y)}{\varepsilon}$ and $\beta_{i}=\beta:=\frac{q}{\varepsilon^{2}}, i=1,2, \ldots, k, q=\left(\exp \left(\frac{2 T_{\max }}{n}\right)-1\right)^{-1}$ then repeating the proof of Lemma 2 we can show that any solution $s(t)$ of the system (8)-(9) satisfies the inequality $\|s(t)\|_{\infty} \leq \varepsilon, \forall t \geq T_{\max }$.

By analogy with single input case let us show now that there exist polynomials $p_{i} \in \mathbb{P}^{i}, i=$ $1,2, \ldots, k$ with nonnegative coefficients such that $\left\|y_{i}(t)\right\|_{\infty} \leq p_{i}(q) \varepsilon$ for $\forall t>T_{\max }$, where $y(t)=\left(y_{1}(t), \ldots, y_{k}(t)\right)^{T}$ is an arbitrary solution of the system (7), (9).

Denote the vector-modulus and the matrix-modulus operation by $\llbracket \cdot \llbracket$, i.e. $\llbracket v \rrbracket=\left(\left|v_{1}\right|, \ldots,\left|v_{r}\right|\right)^{T}$ for the vector $v=\left(v_{1}, \ldots, v_{r}\right)^{T}$ and $\llbracket W \rrbracket:=\left\{\left|w_{i j}\right|\right\}$ for the matrix $W=\left\{w_{i j}\right\}$. The inequalities $\llbracket v_{1} \rrbracket \preceq \rrbracket v_{2} \rrbracket$ and $\rrbracket W_{1} \rrbracket \preceq \rrbracket W_{2} \rrbracket$ are understood in a component-wise sense.

Let $\tilde{\psi}_{1}=0, \tilde{\psi}_{i+1}=\llbracket A_{i+1}^{+} \rrbracket\left(\alpha_{i} z_{i}+\beta z_{i}^{3}+\sum_{j=1}^{i} \llbracket A_{i j} \rrbracket\left(z_{j}+\tilde{\psi}_{j}\right)+\sum_{k=1}^{i} \frac{\partial \tilde{\psi}_{i}}{\partial z_{k}}\left(\alpha_{k} z_{k}+\beta z_{k}^{3}+\llbracket A_{i i+1} \rrbracket z_{k+1}\right)\right)$, where $z_{i} \in \mathbb{R}_{+}^{n_{i}}, i=1,2, \ldots, k$. Following the proof of Corollary 2 we can show that $\llbracket \psi_{i}\left(s_{1}, \ldots, s_{i}\right) \rrbracket \preceq$ $\tilde{\psi}_{i}\left(\llbracket s_{1} \rrbracket, \ldots, \rrbracket s_{i} \rrbracket\right), \tilde{\psi}_{i}\left(\varepsilon \bar{e}_{n_{1}}, \ldots, \varepsilon \bar{e}_{n_{i}}\right)=\varepsilon \sum_{j=0}^{i}\left(\beta \varepsilon^{2}\right)^{j} M_{i j} \bar{e}_{n_{j}}$, where $\bar{e}_{r}=(1, \ldots, 1)^{T} \in \mathbb{R}_{+}^{r}$ and $M_{i j} \in$ $\mathbb{R}^{n_{i} \times n_{j}}$ are matrices with nonnegative elements. Hence it directly follows that $\left\|y_{i}\right\|_{\infty} \leq\left\|s_{i}\right\|_{\infty}+$ $\left\|\psi_{i}\left(s_{1}, \ldots, s_{i}\right)\right\|_{\infty} \leq \varepsilon+\left\|\tilde{\psi}_{i}\left(\varepsilon \bar{e}_{n_{1}}, \ldots, \varepsilon \bar{e}_{n_{i}}\right)\right\|_{\infty}=\varepsilon+\varepsilon \sum_{j=0}^{i}\left(\beta \varepsilon^{2}\right)^{j}\left\|M_{i j}\right\|_{\infty}:=p_{i}(q) \varepsilon$.

Similarly to single input case the polynomials $p_{i}(q)$ can be calculated using some symbolic computation package. For instance, $p_{1}(q)=1$ and $p_{2}(q)=1+\left\|A_{12}^{+}\right\|_{\infty}\left(1+\left\|A_{12}\right\|_{\infty}+q+\left\|A_{11}\right\|_{\infty}\right)$. Therefore, we have just proven the main theorem for the multi input case.

Theorem 3. If the control $u$ has the form (9) with parameters $\alpha_{i}=1+\left\|A_{i i+1}\right\|_{\infty}, i=1,2, \ldots, k-$ $1, \alpha_{k}=1+\frac{\gamma_{0}\left\|A_{k k+1}\right\|_{\infty}\|G\|_{1} p_{k}(q)}{r}, \beta_{i}=\frac{q\|G\|_{1}^{2} p_{k}^{2}(q)}{r^{2}}, i=1,2, \ldots, k, q=\left(\exp \left(\frac{2 T_{\max }}{n}\right)-1\right)^{-1}$, where $r>0$ and $T_{\max }>0$ are arbitrary positive numbers, then the ball $B_{r}$ is the globally fixed-time attractive set of the closed-loop system (2) with the settling-time function bounded by $T_{\max }$. 


\section{Fixed-time stability}

In this subsection we assume $\operatorname{rank}[B, A B]=n$. Then Algorithm 1 terminates after the first recursive step, so the transformation $y=G x$ brings the original system to the form

$$
\left\{\begin{array}{l}
\dot{y}_{1}=A_{11} y_{1}+A_{12} y_{2} \\
\dot{y}_{2}=A_{21} y_{1}+A_{22} y_{2}+A_{23}(u+\gamma)
\end{array}\right.
$$

where $y_{1} \in \mathbb{R}^{n_{1}}, y_{2} \in \mathbb{R}^{n_{2}}, n_{2}=\operatorname{rank}(B), n_{1}=n-n_{2}, \operatorname{rank}\left(A_{12}\right)=n_{1} \leq \operatorname{rank}\left(A_{23}\right)=n_{2}$. Denote $A_{12}^{\perp}=\operatorname{null}\left(A_{12}\right)^{T}$.

Theorem 4. Let the controller $u(t, y), y=\left(y_{1}^{T}, y_{2}^{T}\right)^{T} \in \mathbb{R}^{n}$ has the form:

$$
u=A_{23}^{+}\left(u_{e q}(y)+u_{d}(t, y)+u_{p}(y)\right),
$$

$$
\text { where } \begin{aligned}
u_{e q} & =-A_{12}^{+}\left(\left(A_{11}^{2}+A_{12} A_{21}\right) y_{1}+\left(A_{11} A_{12}+A_{12} A_{22}\right) y_{2}\right)-\left(A_{12}^{\perp}\right)^{+} A_{12}^{\perp}\left(A_{21} y_{1}+A_{22} y_{2}\right), \\
& u_{d}=-\frac{\alpha_{1}+3 \beta_{1}\left\|y_{1}\right\|_{\infty}^{2}+2\left\|A_{12} A_{23}\right\|_{\infty} \gamma_{0}(t, y)}{2} A_{12}^{+} \operatorname{sign}\left[s_{1}\right]-\gamma_{0}(t, y)\left\|A_{12}^{\perp} A_{23}\right\|_{\infty}\left(A_{12}^{\perp}\right)^{+} \operatorname{sign}\left[s_{2}\right], \\
& u_{p}=-A_{12}^{+}\left(\alpha_{2} s_{1}+\beta_{2} s_{1}^{[3]}\right)^{\left[\frac{1}{2}\right]}-\left(A_{12}^{\perp}\right)^{+}\left(\alpha_{3} s_{2}+\beta_{3} s_{2}^{[3]}\right)^{\left[\frac{1}{2}\right]}, \\
& s_{1}=A_{11} y_{1}+A_{12} y_{2}+\left(\left(A_{11} y_{1}+A_{12} y_{2}\right)^{[2]}+\alpha_{1} y_{1}+\beta_{1} y_{1}^{[3]}\right)^{\left[\frac{1}{2}\right]}, \quad s_{2}=A_{12}^{\perp} y_{2}
\end{aligned}
$$

with $0.5 \alpha_{1}=\alpha_{2}=4 \alpha_{3}=0.5 \beta_{1}=\beta_{2}=4 \beta_{3}=64 T_{\max }^{-2}, T_{\max }>0$. Then the origin of the closed-loop system (10) is globally fixed-time stable with the settling-time function bounded by $T_{\max }$.

Proof: First of all remark that $A_{12}\left(A_{12}^{\perp}\right)^{+}=0$ and $A_{12}^{\perp} A_{12}^{+}=0$. Denote $z=A_{11} y_{1}+A_{12} y_{2}$. In this case the system (10) can be rewritten in the form

$$
\dot{y}_{1}=z, \quad \dot{z}=-A_{12} u_{d}-A_{12} u_{q}+A_{12} A_{23} \gamma, \quad \dot{s}_{2}=-A_{12}^{\perp} u_{d}-A_{12}^{\perp} u_{p}+A_{12}^{\perp} A_{23} \gamma
$$

and $s_{1}=z+\left(z^{[2]}+\alpha_{1} y_{1}+\beta_{1} y_{1}^{[3]}\right)^{\left[\frac{1}{2}\right]}$. Following the proof of Theorem 2 we similarly show that $y_{1}(t)=0$ and $z(t)=0$ for all $t \geq T_{\max }$. Hence $A_{12} y_{2}(t)=0$ for all $t \geq T_{\max }$. Taking into account formulas for $u_{d}$ and $u_{p}$ we obtain $\dot{s}_{2}=-\gamma_{0}(t, y)\left\|A_{12}^{\perp} A_{23}\right\|_{\infty} \operatorname{sign}\left[s_{2}\right]-\left(\alpha_{3} s_{2}+\beta_{3} s_{2}^{[3]}\right)^{\left[\frac{1}{2}\right]}+A_{12}^{\perp} A_{23} \gamma$. Hence it can be easily shown that $s_{2}(t)=A_{12}^{\perp} y_{2}(t)=0$ for all $t \geq T_{\max }$.

Theorem 4 extends the "nested" second order sliding mode control and the fixed-time controller presented in Theorem 2 to the multi input case.

Remark that the control (11) has the form closed to classical sliding mode controllers [24]. The equivalent control term $u_{e q}$ compensates the inner dynamic of the system, the switching term $u_{d}$ ensures the sliding mode existence and the new additional term $u_{p}$ has the form required for fixed-time stability.

For $n_{1}=n_{2}$ we have $\operatorname{ker}\left(A_{12}\right)=\emptyset$, so the terms with $A_{12}^{\perp}$ and $\left(A_{12}^{\perp}\right)^{+}$presented in (11) disappear. 


\section{NumERICAL EXAMPLES}

Consider as a benchmark example the system (2) with $A=\left(\begin{array}{ccc}1 & -3 & 2 \\ -2 & 0 & 3 \\ 0 & -1 & 4\end{array}\right), B=\left(\begin{array}{cc}2 & 0 \\ -1 & 1 \\ 0 & -3\end{array}\right), f=\left(\begin{array}{c}2 \\ 0 \\ -3\end{array}\right) \sin (t), G=\left(\begin{array}{ccc}0.4286 & 0.8571 & 0.2857 \\ -0.8571 & 0.4857 & -0.1714 \\ -0.2857 & -0.1714 & 0.9429\end{array}\right)$. The transformation $y=G x$ brings the system to the block from (10) with $y_{1} \in \mathbb{R}, y_{2} \in \mathbb{R}^{2}$, $A_{11}=-0.5918, A_{23}=\left(\begin{array}{cc}-2.2 & 1.0 \\ -0.4 & -3.0\end{array}\right), A_{21}=\left(\begin{array}{c}1.2980 \\ 0.7184\end{array}\right), A_{22}=\left(\begin{array}{cc}3.0612 & -0.8367 \\ -0.5510 & 2.5306\end{array}\right)$, $A_{12}=\left(\begin{array}{ll}-0.4449 & 4.9469\end{array}\right)$. For this case we have $\gamma(t, x)=(\sin (t), \sin (t))^{T}$ and $\gamma_{0}(t, x)=1$.

\section{A. Example of fixed-time attractivity}

Let $T_{\max }=6, r=1$. Following Theorem 3 we select the controller $u(t, x)$ in the form (9) with the control parameters $\alpha_{1}=6.3918, \alpha_{2}=13.333, \beta_{1}=\beta_{2}=0.0327$. Fig.1(a) and Fig. 1(b) show evolutions of the system states for $x(0)=(1,0,0)$ and $x(0)=(10,0,0)$. Fig. 1 (c) presents the plot for the control magnitude. Numerical experiments show that the predetermined settling time

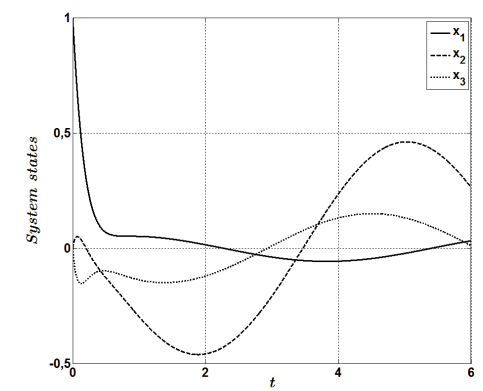

(a) $x(0)=(1,0,0)$

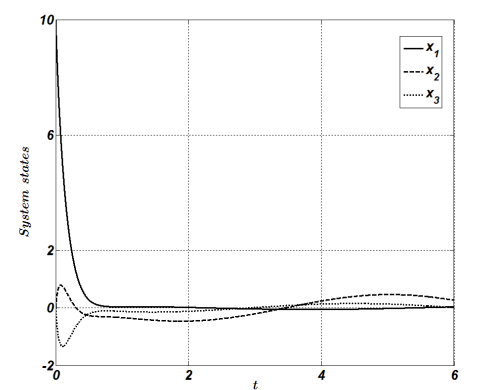

(b) $x(0)=(10,0,0)$

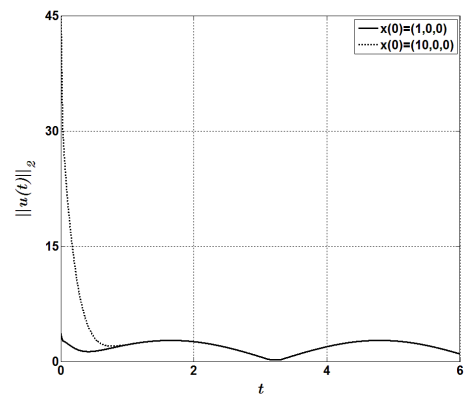

(c) Control magnitude.

Fig. 1. Simulation results for fixed-time attraction.

estimate $T_{\max }=6$ is too conservative. Obtained convergence times to the ball $B_{1}$ are less than 1 .

\section{B. Example of fixed-time stability}

According to Theorem 4 the controller of the form (11) with parameters $\alpha_{1}=\beta_{1}=1, \alpha_{2}=\beta_{2}=0.5$, $\alpha_{3}=\beta_{3}=0.25$ provides fixed-time stability of the considered system guaranteing the global settling-time estimate $T_{\max }=8$. Simulations results approve declared property (see Fig. 2 ). 


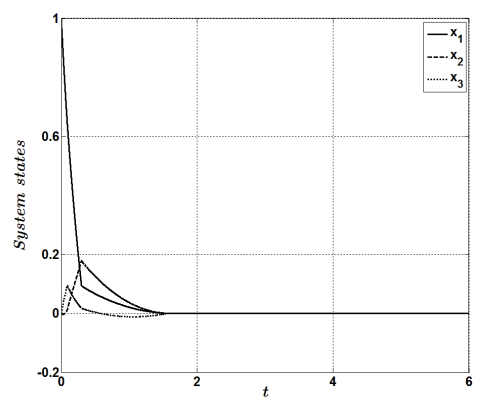

(a) $x(0)=(1,0,0)$

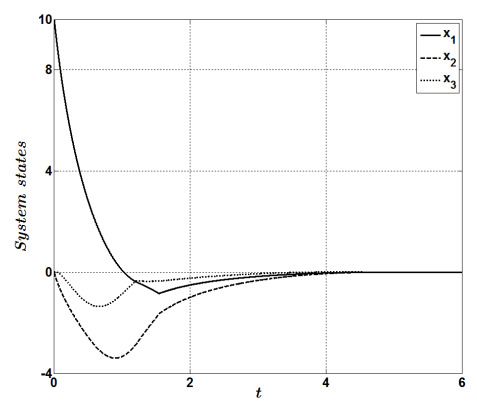

(b) $x(0)=(10,0,0)$

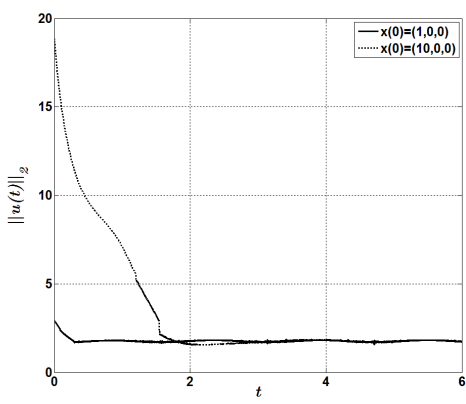

(c) Control magnitude.

Fig. 2. Simulation results for fixed-time stabilization.

In contrast to polynomial feedback numerical simulations for the fixed-time stabilizing controller demonstrates very small conservatism in settling-time estimate showing that for $x(0)=$ $\left(10^{7}, 0,0\right)$ the settling-time is equal approximately to 7.2 .

\section{CONCLUSIONS}

In this paper the new control problems are considered. They consist in designing of the feedback control for linear uncertain plant that guarantees convergence of system trajectories to the origin or into selected neighborhood of the origin in predetermined finite time independently on initial conditions. The described property is called in this paper by fixed-time stability and attractivity, respectively. Controllers that provides fixed-time attractivity to the closed-loop systems are designed in the form of polynomial feedbacks under the classical assumption on controllability of the pair $(A, B)$. Fixed-time stabilizing controllers presented for more restrictive case $\operatorname{rank}[B, A B]=n$ are modification of the second order sliding mode control algorithm. It is proven that all controllers are robust with respect to matched uncertainties and disturbances.

Numerical experiments approve the declared fixed-time properties for the closed-loop system with the proposed control algorithms. However, they show that for polynomial feedbacks the real convergence time is less than the predetermined. This fact probably points out ineffective usage of the control resource. So, the problem of optimal selection of the control parameters in order to minimize the control energy and magnitude appears. But this is another story.

\section{ACKNOWLEDGEMENTS}

The author wishes to thank professor Boris T. Polyak for helpful discussions and remarks. 


\section{REFERENCES}

[1] V. Andrieu, L. Praly, A. Astolfi Homogeneous Approximation, Recursive Obsertver and Output Feedback, SIAM Journal of Control and Optimization, 47(4):1814-1850, 2008.

[2] G. Bartolini, A. Pisano, E. Punta, E. Usai, A survey of applications of second-order sliding mode control to mechanical systems, International Journal of Control, 76(9/10):875-892, 2003.

[3] F. J. Bejarano \& L. M. Fridman, High order sliding mode observer for linear systems with unbounded unknown inputs, International Journal of Control, 9:1920-1929, 2010.

[4] S.P. Bhat, D.S. Bernstein, Finite-time stability of continuous autonomous systems, SIAM Journal of Control and Optimization, 38(3):751-766, 2000.

[5] F.L. Chernousko, I.M. Ananevskii, S.A. Reshmin, Control of Nonlinear Dynamical Systems:Methods and Applications, Berlin: Springer-Verlag, 2008.

[6] E. Cruz-Zavala, J.A. Moreno, L. Fridman, Uniform Second-Order Sliding Mode Observer for Mechanical Systems, International Workshop on Variable Structure Systems,14-19,2010.

[7] S.V. Drakunov et al., Block Control Principle I, Automation and Remote Control, 51(5):601-609, 1990.

[8] C. Ebenbauer, F. Allgöwer, Analysis and design of polynomial control systems using dissipation inequalities and sum of squares, Computers and Chemical Engineering, 30:1590-1602, 2006.

[9] D. Efimov, Uniting global and local controllers under acting disturbances, Automatica, 42:489-495, 2006.

[10] R. Engel, G. Kreisselmeier, A Continuous-Time Observer Which Converges in Finite Time, IEEE Transactions on Automatic Control, 47:12021204, 2002.

[11] A. Ferrara \& L. Giacomini, On multi-input backstepping design with second order sliding modes for a class of uncertain nonlinear systems, International Journal of Control, 71(5):767-788, 1998.

[12] A.F. Filippov, Differential Equations with Discontinuous Right-Hand Sides. Kluwer Academic Publishers, 1988.

[13] V. T. Haimo, Finite time controllers. SIAM Journal of Control and Optimization, 24(4):760-770, 1986.

[14] Y. Hong, Z.-P. Jiang, Finite-Time Stabilization of Nonlinear Systems With Parametric and Dynamic Uncertainties IEEE Transactions on Automatic Control, 51(12):1950-1956, 2006

[15] S. A. Krasnova, T. G. Sirotina, V.A. Utkin, A structural approach to robust control, Automation and Remote Control, 72(8):1639-1666, 2011.

[16] A. Levant, Homogeneity approach to high-order sliding mode design. Automatica, 41:823-830, 2005.

[17] A. Levant, Principles of 2-sliding mode design, Automatica, 43:576-586, 2007.

[18] M. James, Finite Time Observers and Observability, Conference on Decision and Control, 770-771, 1990.

[19] E. Moulay, W. Perruquetti, Finite-time stability and stabilization: state of the art, Lecture Notes in Control and Information Sciences, 334:23-41, 2006.

[20] Y. Orlov, Finite Time Stability and Robust Control Synthesis of Uncertain Switched Systems. SIAM Journal of Control and Optimization, 43(4):1253-1271, 2005.

[21] Y. Orlov, Discontinous systems: Lyapunov analysis and robust synthesis under uncertainty conditions. Springer-Verlag,2009.

[22] A. Polyakov, A. Poznyak, Lyapunov function design for finite-time convergence analysis: "twisting" controller for second order sliding mode realization. Automatica, 45(2):444-448, 2009.

[23] T. Raff, F. Allgöwer, An Observer that Converges in Finite Time Due to Measurement-based State Updates, IFAC World Congress, 2693-2695, 2008.

[24] V.I. Utkin, Sliding Modes in Control Optimization. Springer Verlag, Berlin, 1992. 